\title{
The Role of Oxidized Low-Density Lipoproteins in Atherosclerosis: The Myths and the Facts
}

\author{
Giuseppe Maiolino, Giacomo Rossitto, Paola Caielli, Valeria Bisogni, \\ Gian Paolo Rossi, and Lorenzo A. Calò \\ Department of Medicine (DIMED), Internal Medicine 4, University of Padova, Via Giustiniani 2, 35128 Padova, Italy \\ Correspondence should be addressed to Lorenzo A. Calò; renzcalo@unipd.it
}

Received 10 June 2013; Accepted 28 August 2013

Academic Editor: Ishak Tekin

Copyright (c) 2013 Giuseppe Maiolino et al. This is an open access article distributed under the Creative Commons Attribution License, which permits unrestricted use, distribution, and reproduction in any medium, provided the original work is properly cited.

\begin{abstract}
The oxidative modification hypothesis of atherosclerosis, which assigns to oxidized low-density lipoproteins (LDLs) a crucial role in atherosclerosis initiation and progression, is still debated. This review examines the role played by oxidized LDLs in atherogenesis taking into account data derived by studies based on molecular and clinical approaches. Experimental data carried out in cellular lines and animal models of atherosclerosis support the proatherogenic role of oxidized LDLs: (a) through chemotactic and proliferating actions on monocytes/macrophages, inciting their transformation into foam cells; (b) through stimulation of smooth muscle cells (SMCs) recruitment and proliferation in the tunica intima; (c) through eliciting endothelial cells, SMCs, and macrophages apoptosis with ensuing necrotic core development. Moreover, most of the experimental data on atherosclerosis-prone animals benefiting from antioxidant treatment points towards a link between oxidative stress and atherosclerosis. The evidence coming from cohort studies demonstrating an association between oxidized LDLs and cardiovascular events, notwithstanding some discrepancies, seems to point towards a role of oxidized LDLs in atherosclerotic plaque development and destabilization. Finally, the results of randomized clinical trials employing antioxidants completed up to date, despite demonstrating no benefits in healthy populations, suggest a benefit in high-risk patients. In conclusion, available data seem to validate the oxidative modification hypothesis of atherosclerosis, although additional proofs are still needed.
\end{abstract}

\section{Introduction}

Recent postulates on atherosclerosis designate the appearance of qualitative changes on endothelial cells, triggered by "irritative" stimuli (e.g., hypertension, dyslipidemia, and cigarette smoking), as an early pathogenic event [1]. This process occurs at specific segments of the arterial tree, mainly branching points and bifurcations, characterized by disturbed laminar blood flow, probably owing to differences in arteries regional development [2] and to the loss of the atheroprotective effect of laminar shear stress [3]. In this setting, the endothelium expresses adhesion and chemotactic molecules and acquires an increased permeability to macromolecules, which modifies the composition of the subendothelial extracellular matrix. Hence, the entry of low-density lipoprotein (LDL) particles in the arterial wall followed by their retention through the binding of apolipoprotein B100 to proteoglycans of the extracellular matrix [4] is held to be a key-initiating factor in early atherogenesis [5]. The LDL particles trapped in the subintimal extracellular matrix are mildly oxidized by resident vascular cells [6]. They retain the capability of binding to the $\operatorname{LDL}$ receptor $[6,7]$ and to exert their proatherogenic effects [8-10], including stimulation of the resident vascular cells to produce monocyte chemotactic protein-1, granulocyte, and macrophage colony-stimulating factors. These molecules promote monocytes recruitment and their differentiation into macrophages, which are able to further promote the oxidation of LDLs [11] through myeloperoxidase and reactive oxygen species. Completely oxidized LDLs, characterized by an increased apolipoprotein B100 negative charge, are recognized by scavenger receptors on macrophages and internalized to form foam cells [12], the hallmark of the atherosclerotic lesion. Furthermore, macrophages play a key role in atherogenesis 


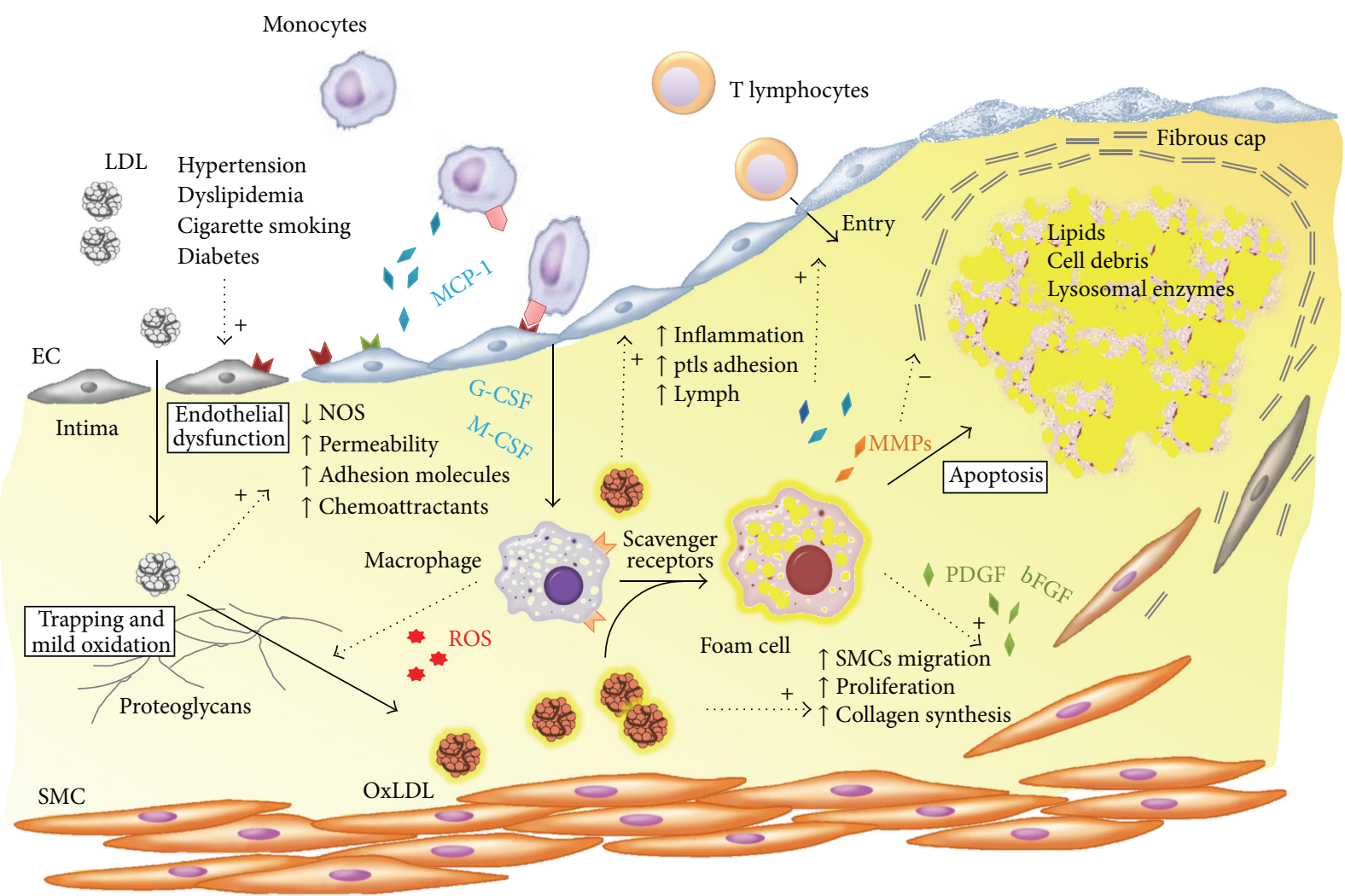

FIGURE 1: Putative pathway of oxidized low-density lipoprotein (oxLDL) in the atherogenetic process according to the oxidative hypothesis of atherosclerosis.

through their proinflammatory action, which involves the production of interleukin- $\beta$ and tumor necrosis factor (Figure 1).

Other main effectors in the development of atherosclerotic lesions are smooth muscle cells (SMCs), which are recruited from the tunica media to the subendothelial space, where they proliferate in response to mediators such as the platelet-derived growth factor. SMCs residing in the tunica intima produce extracellular matrix molecules, for example, interstitial collagen and elastin, and build the fibrous cap that overlies the growing atherosclerotic plaque. The latter entails macrophage-derived foam cells, cellular debris, and extracellular lipids, which are inefficiently cleared due to defective efferocytosis and thereby form the so-called necrotic core of the plaque [13].

The atherosclerotic plaque becomes clinically manifest when it reaches an advanced stage due to its blood flowlimiting effects or its destabilization with ensuing thrombosis. Unfortunately, the latter complication, which is responsible for ischemic events, is not strictly related to the degree of stenosis at angiography $[14,15]$ as its occurrence stands mostly on the cellular features of the plaque and particularly on the thickness of the overlying fibrous cap [16, 17]. In fact, atherosclerotic plaques prone to rupture are characterized by accumulation of inflammatory cells, mostly at the shoulder regions. These cells degrade collagen through release of collagenolytic enzymes, mainly matrix metalloproteinases
(MMPs), and also reduce its synthesis by inducing SMCs apoptosis [18].

Many excellent reviews on the current knowledge of atherosclerosis are available, but few are focused on oxidized LDLs. Hence, this review examines the role played by oxidized LDLs in atherogenesis taking into account data derived by studies based on molecular and clinical approaches.

\section{Evidence Linking Oxidized LDLs to Atherosclerosis}

The oxidative modification hypothesis designates the oxidative change of LDLs as a crucial, if not mandatory, step in atherogenesis [19]. This theory originated from studies demonstrating that LDLs modified by endothelial cells, transformation entailing an oxidation process [20], could be internalized and accumulated avidly by macrophages [21, 22], leading to foam cell formation, although these cells could also be generated from macrophages internalizing native LDLs from the medium through micropinocytosis [23], or by uptake of aggregated LDLs or LDL immune complexes.

Several potential mechanisms can explain how LDL oxidative modification occurs within the arterial wall in vivo. A major role has been proposed for the 12/15-lipoxygenase $[24,25]$ because $(1)$ it is expressed in atherosclerotic plaques but not in normal vessels [26] and (2) its inhibition was 
associated with decreased oxidation of LDLs [27] and reduced atherosclerosis in animal models [25, 28, 29]. Myeloperoxidase, a heme enzyme secreted by neutrophils and monocytes/macrophages, is another suggested agent. It was found in human atherosclerotic plaques [30] and modifies LDLs, thus increasing their affinity for CD36 and SR-A [31, 32], the scavenger receptors mediating the uptake of oxidized LDLs by macrophages. Nitric oxide synthase (NOS) and nicotinamide adenine dinucleotide phosphate (NADPH)-oxidase are other putative players as their products nitric oxide and superoxide anion, respectively, can combine to form the strong oxidizing agent peroxynitrite.

Native LDLs are internalized by macrophages at a pace too low to account for foam cells formation [33] owing to LDL receptor downregulation. Oxidative modification of LDLs increases their uptake by macrophages [20], via scavenger receptors. The latter are not downregulated in response to increased intracellular cholesterol, which explains why foam cells formation is held to occur with oxidized LDLs and not with native LDLs.

Besides contributing to the formation of lipid-laden macrophages, oxidized LDLs exhibit a wide array of biological properties, which are deemed to promote atherosclerosis.

(i) Oxidized LDLs exert chemotactic activity for monocytes [34], stimulate their binding to endothelial cells [35] by inducing the expression of intercellular adhesion molecule-1 and vascular-cell adhesion molecule1 [36], are mitogenic for macrophages [37], and promote their trapping in the intima, while limiting their egress from the arterial wall [38]. Hence, oxLDL is key for recruitment, activation, and proliferation of monocytes/macrophages in the arterial wall.

(ii) Oxidized LDLs increase the expression of growth factors, such as platelet-derived growth factor (PDGF) and basic fibroblast growth factor (FGF) by endothelial cells and macrophages. The former stimulates migration of SMCs [39-41], while the latter induces SMCs proliferation [42].

(iii) Oxidized LDLs stimulate collagen production by SMCs [43], thus contributing to the fibrous cap lining the atherosclerotic plaque and the expansion of the lesion size. However, they could also promote fibrous cap thinning by increasing secretion of matrix metalloproteinase 1 [44] and matrix metalloproteinase 9, decreasing production of the tissue inhibitor of metalloproteinase 1 [45], and inducing SMCs apoptosis [46]. Therefore, they can contribute to the occurrence of vulnerable plaques [16, 17]. Hence, taken together, this evidence involved oxidized LDLs in the progression of the atherosclerotic plaque and the development of its complications.

(iv) Oxidized LDLs are cytotoxic to vascular cells [47, 48] and promote their apoptosis $[49,50]$ with ensuing release in the subendothelial space of lipids and lysosomal enzymes, enhancing the progression of the atherosclerotic plaque [47] and the production of the necrotic core. (v) Oxidized LDLs stimulate platelet adhesion and aggregation, by decreasing endothelial production of nitric oxide, increasing prostacyclin production [51, 52], and stimulating the synthesis of prostaglandins and prostaglandin precursors [53]. Moreover, they decrease the secretion of the tissue-type plasminogen activator and increase that of plasminogen activator inhibitor-1 followed by a reduction of the fibrinolytic activity of endothelium [54-56]. Ultimately, they may determine vasoconstriction by inhibiting nitric oxide [57] and increasing endothelin production [58]. Taken together, these findings may explain the thrombotic complications of advanced atherosclerotic plaques.

\section{In Vivo Models Supporting the Oxidized LDL Role in Atherosclerosis}

Several studies were carried out in vivo in animal models where either a modulation of oxidative stress or manipulation of the scavenger receptor was undertaken, in order to prove the role of oxidized LDLs in the pathogenesis of atherosclerosis.

In an animal model of increased oxidative stress obtained through the overexpression of 15-lipoxygenase in the vascular wall, larger atherosclerotic lesions were found in LDL receptor-deficient mice [59]. However, a decreased atherosclerosis in cholesterol-fed rabbits and WHHL rabbits whose macrophages overexpressed human 15-lipoxygenase was also reported [60]. Animal models of reduced oxidative stress, instead, were obtained through knockout of oxidative stress-related genes or increasing the antioxidants: in three different knockout mouse models for 12/15-lipoxygenase, a decreased severity of atherosclerosis was seen $[25,61-$ 64]. However, in apoE-deficient mice, the knocking out of the macrophage-specific 12/15-lipoxygenase increased the extension of atherosclerotic lesions [65].

The knockout in atherosclerosis-prone mice models of either SR-A or CD36 scavenger receptors, accounting for almost $90 \%$ of macrophage oxidized LDLs uptake [66], was demonstrated to be efficacious in decreasing the atherosclerotic burden $[67,68]$. However, these results were not confirmed in another mice model with a CD36 and SR-A double knockout [69].

The results of these studies proved to be highly contradictory, due to the different animal models used, the different genetic background, and the unexpected consequences of gene deletions [70].

Finally, in spite of these conflicting data, support to the oxidative theory comes from extensive literature on the treatment of atherosclerosis-prone animals with antioxidants (reviewed by Witztum and Steinberg [71]). Most of these studies were carried out with probucol and demonstrated a protection from atherosclerotic lesions with the exception of the murine models, possibly secondary to a peculiar toxicity of this molecule in mice. In fact, in apoE-deficient mice fed with vitamin $\mathrm{E}$, decreased atherosclerosis, paralleled with a decrease of aortic wall, plasma, and urinary $\mathrm{F}_{2}$ isoprostanes, a marker of oxidative stress, was observed [72]. 


\section{Human Findings Supporting the Oxidized LDLs Role in Atherosclerosis}

There is a wealth of literature on the association between oxidized LDLs and cardiovascular events. An important premise needs to be made beforehand, however, in that oxidation of LDLs induces immunogenic epitopes in their particles [73] with ensuing generation of antibodies against them (oxLDL Abs). Since these autoantibodies are detectable in the sera of the majority of patients with advanced atherosclerotic lesions [74], they can be viewed as in vivo markers of LDL oxidation. Oxidized LDLs and their involvement in atherosclerosis can therefore be assessed by two ways: (1) using murine monoclonal antibodies directed toward different oxidized LDLs epitopes and (2) determining the immunogenic response to oxidized LDLs. Both approaches have advantages and pitfalls, as reviewed in detail by Tsimikas [75].

Human studies on the association of oxidized LDLs with atherosclerosis or cardiovascular events have been highly conflicting (for rev. [76]). Some cross-sectional studies suggested a direct association of oxidized LDLs or oxLDL Abs with atherosclerosis in different vascular beds [74, 77, 78], whereas others found no association with coronary atherosclerotic burden in coronary artery disease patients [79-81]. Owing to these contradictory results, we focused on cohort studies, which are more solid and less prone to serendipitous findings, because of a lower chance for selection and recall bias [82].

Among the twenty-two cohort studies reporting on cardiovascular events, fourteen were positive [81, 83-94] (Table 1) and eight were negative [80, 95-101] (Table 2). Due to potential publication bias, the preponderance of positive results clearly does not provide a proof of the strength of the association [102]. However, it is important to highlight that three $[95,99,100]$ out of eight negative studies were completed in healthy people. This carries a limitation in that the robustness of cohort studies depends on the assumption that the control group-in this case those exposed to low levels of oxidized LDLs-has features as close as possible to the group exposed to high levels of oxidized LDLs [103, 104]. Theoretically, this goal can be better accomplished in selected populations made of patients with similar risk profile, rather than in studies recruiting healthy persons. Among these negative studies, the first one reported on cardiovascular events in a large cohort of more than three thousand elderly patients who had 420 cardiovascular events after 5 years of followup [95]. Oxidized LDLs were predictive of cardiovascular events only if a multivariate analysis was not adjusted for the presence of metabolic syndrome. In the second study, which enrolled almost three thousand healthy subjects, malonyldialdehyde-LDL autoantibodies were not associated with cardiovascular events [99]. In the latter, similarly performed in a healthy population, malonyldialdehydeand $\mathrm{Cu}-\mathrm{LDL}$ autoantibodies and oxidized LDLs were not predictive of progression of carotid atherosclerosis [100].

The lack of association between oxidized LDLs and cardiovascular events, possibly due to lack of statistical power, was also reported in two small cohorts of high-risk end-stage renal disease [101] and diabetes mellitus patients [96].
Other negative studies enrolling patients with coronary heart disease $[80,97,98]$ were either too small $[80,97]$ and with a number of cardiovascular events too low to allow detection of any effect of oxidized LDLs or had an endpoint not appropriate to study atherosclerosis because most of the cardiovascular events were coronary artery restenosis (75\% of total events) [98]. Moreover, it is worth highlighting that the negative study published by Tsimikas et al. [80] was on the same cohort where an association between coronary artery atherosclerosis and oxidized LDLs was demonstrated [78].

Among the positive studies four out of fourteen were carried out in a healthy cohort $[88,90,92,94]$, thus exposing them to the same considerations expressed above. Moreover, it has to be pointed out that three of these studies were completed in the same cohort, the Brunick study, at different time points of follow up, that is, 5 [90], 10 [92], and 15 years [94], and all demonstrated a predictive value of oxidized LDLs on cardiovascular events, contradicting the results on carotid artery atherosclerosis [100] on the same population.

Other studies demonstrating an association between oxidized LDLs and cardiovascular events enrolled small cohorts of either high-risk patients [83-86] or coronary artery disease patients $[87,89,105]$. Therefore these positive results could be secondary to serendipitous findings, as suggested by the opposite results on similar cohorts of end-stage renal failure patients where high oxLDL Abs titer was associated to either low [84] or high [86] cardiovascular mortality.

Two studies reported an association of oxidized LDLs with cardiovascular events in diabetics [93] and acute coronary syndrome patients [91]. Finally, using a prospective cohort study design and an unequivocal definition of the coronary artery disease phenotype, we reported the association of oxLDL Abs with cardiovascular mortality and cardiovascular events in more than 700 coronary artery disease patients [81].

In conclusion, most cohort studies reported an association between oxidized LDLs and cardiovascular events or mortality, in particular those including either a very high-risk population, that is, with end-stage renal disease and diabetes, or coronary artery disease patients. However, despite being an appealing hypothesis, the oxidation theory of atherosclerosis is not conclusively corroborated by observational studies, which have conflicting results, probably owing to the enrolment selection criteria and low statistical power.

\section{Clinical Trials on Antioxidants and the Oxidized LDL Hypothesis}

The oxidative theory of atherosclerosis would be conclusively proven by the beneficial effects of oxidative stress decrease on cardiovascular events. Therefore, the analysis of controlled randomized trials on antioxidant therapy in this setting is crucial. The first report on efficacy of antioxidants on cardiovascular events in coronary artery disease patients [106] was later confirmed by further studies [107-110] (Table 3), but numerous subsequent randomized clinical trials failed to prove any benefit [111-126] (Table 4). Moreover, metaanalyses on this issue are discordant $[127,128]$. 
TABLE 1: Cohort studies demonstrating an association between oxidized low-density lipoprotein measurement and cardiovascular events.

\begin{tabular}{|c|c|c|c|c|c|c|}
\hline Oxidative oxLDL test & $\begin{array}{l}\text { Population } \\
\text { under study }\end{array}$ & CV endpoints & $\begin{array}{l}\text { Number of } \\
\text { events }\end{array}$ & Followup & Findings & Reference \\
\hline OxLDL Abs 4E06 & 326 men & IMT & na & 3 years & $\begin{array}{l}\text { OxLDL predicted IMT and carotid } \\
\text { plaque progression }\end{array}$ & $\begin{array}{l}\text { Wallenfeldt et } \\
\text { al. [88] }\end{array}$ \\
\hline OxLDL Abs 4E06 & 765 subjects & $\mathrm{CV}$ events & $77 \mathrm{CV}$ events & 5 years & OxLDL predicted CV events & $\begin{array}{c}\text { Tsimikas et al. } \\
{[90]}\end{array}$ \\
\hline OxLDL Abs 4E06 & 765 subjects & CV events & $82 \mathrm{CV}$ events & 10 years & OxLDL predicted CV events & $\begin{array}{c}\text { Kiechl et al. } \\
{[92]}\end{array}$ \\
\hline $\begin{array}{l}\text { OxPL/apoB, AutoAbs } \\
\text { MDA-/Cu-oxLDL }\end{array}$ & 765 subjects & CV events & $138 \mathrm{CV}$ events & 15 years & $\begin{array}{c}\text { OxPL/apoB predicted CV events } \\
\text { and stroke; AutoAbs predicted CV } \\
\text { events, stroke, and ACS }\end{array}$ & $\begin{array}{c}\text { Tsimikas et al. } \\
{[94]}\end{array}$ \\
\hline MDA-LDL & 907 NIDDM & CV events, $\mathrm{MI}$ & $\begin{array}{c}152 \mathrm{CV} \\
\text { events, } 43 \mathrm{MI}\end{array}$ & 3.7 years & $\begin{array}{l}\text { MDA-LDL predicted MI and CV } \\
\text { events }\end{array}$ & $\begin{array}{c}\text { Lopes-Virella } \\
\text { et al. [93] }\end{array}$ \\
\hline AutoAbs Cu-oxLDL & 249 ESRD & CV mortality & $74 \mathrm{CV}$ deaths & 63 months & AutoAbs predicted CV mortality & Shoji et al. [84] \\
\hline AutoAbs Cu-oxLDL & $\begin{array}{l}94 \text { ESRD on } \\
\text { hemodialysis }\end{array}$ & Total mortality & 32 deaths & 24 months & AutoAbs predicted mortality & $\begin{array}{l}\text { Bayés et al. } \\
{[85]}\end{array}$ \\
\hline AutoAbs Cu-oxLDL & $\begin{array}{l}94 \text { ESRD on } \\
\text { hemodialysis }\end{array}$ & CV mortality & $33 \mathrm{CV}$ deaths & 4 years & AutoAbs predicted CV mortality & $\begin{array}{l}\text { Bayés et al. } \\
{[86]}\end{array}$ \\
\hline OxLDL Abs DLH3 & $\begin{array}{l}246 \text { pts with } \\
\text { coronary } \\
\text { angiography }\end{array}$ & $\begin{array}{l}\text { CV events: } \\
\text { cardiac death, } \\
\text { MI, PTCA, and } \\
\text { CABG }\end{array}$ & $76 \mathrm{CV}$ events & 38 months & OxLDL predicted CV events & $\begin{array}{l}\text { Shimada et al. } \\
\text { [87] }\end{array}$ \\
\hline $\begin{array}{l}\text { AutoAbs } \\
\text { MDA-oxLDL }\end{array}$ & 734 IHD pts & $\begin{array}{l}\text { CV mortality, } \\
\text { MI, ACS, and } \\
\text { CV events }\end{array}$ & $\begin{array}{l}65 \mathrm{CV} \text { deaths, } \\
153 \mathrm{CV} \text { events }\end{array}$ & 7.2 years & $\begin{array}{l}\text { OxLDL predicted CV death and } \\
\text { events }\end{array}$ & $\begin{array}{l}\text { Maiolino et al. } \\
{[81]}\end{array}$ \\
\hline AutoAbs Cu-oxLDL & $\begin{array}{l}74 \text { PTCA pts, } \\
14 \mathrm{ctr}\end{array}$ & Restenosis & 34 restenosis & 6 months & AutoAbs predicted restenosis & Lee et al. [107] \\
\hline OxLDL Abs DLH3 & $\begin{array}{c}102 \text { primary } \\
\text { PTCA pts, } 86 \mathrm{ctr}\end{array}$ & Restenosis & 25 restenosis & 6 months & OxLDL predicted restenosis & $\begin{array}{c}\text { Naruko et al. } \\
{[89]}\end{array}$ \\
\hline OxLDL Abs 4E06 & 433 ACS pts & CV death, MI & $\begin{array}{l}17 \mathrm{CV} \text { deaths, } \\
57 \mathrm{MI}\end{array}$ & 2 years & OxLDL predicted MI & $\begin{array}{c}\text { Johnston et al. } \\
{[91]} \\
\end{array}$ \\
\hline $\begin{array}{l}\text { OxLDL Abs } \\
\text { FOH1a/DLH3 }\end{array}$ & $\begin{array}{l}84 \mathrm{CHF} \text { pts }(\mathrm{EF} \\
<45 \%), 18 \mathrm{ctr}\end{array}$ & $\begin{array}{l}\text { CV death, } \mathrm{CV} \\
\text { hospitalization, } \\
\text { and fatal } \\
\text { arrhythmia }\end{array}$ & $26 \mathrm{CV}$ events & 780 days & OxLDL predicted CV events & $\begin{array}{l}\text { Tsutsui et al. } \\
\qquad[83]\end{array}$ \\
\hline
\end{tabular}

Abs: antibodies; ACS: acute coronary syndrome; AutoAbs: autoantibodies; CABG: coronary artery by-pass surgery; CHF: congestive heart failure; Crt: controls; CV: cardiovascular; ESRD: end-stage renal disease; IHD: ischemic heart disease; IMT: intima-media thickness; MI: myocardial infarction; oxLDL: oxidized low-density lipoproteins; OxPL/apoB: oxidized phospholipids on apolipoprotein B-100; PTCA: percutaneous transluminal coronary angioplasty; pts: patients.

An in depth analysis of these studies, however, highlighted that most of the negative studies were completed in either healthy or high-risk individuals, whereas results of clinical trials completed in patients with cardiovascular disease demonstrated the benefit conferred by antioxidants in some cases [106, 108, 109], with notable exceptions [112, $113,117,125]$. The fact that treatment was likely given to the wrong patients, that is, with very low risk profile, can explain the failure of antioxidants trials in preventing cardiovascular events in the aforementioned negative reports [129].

Moreover, as in cohort studies, positive effects of antioxidants were witnessed in randomized trials enrolling very high-risk populations, as end-stage renal disease patients in hemodialysis, characterized by an increased oxidative stress, possibly secondary to hemolysis and hemoglobin-induced LDL oxidation $[130,131]$. In these patients, with vitamin E supplementation, as tested in the SPACE trial randomizing patients to vitamin $\mathrm{E}$ or placebo [108], cardiovascular events were reduced by $54 \%$ and myocardial infarction by $70 \%$. Accordingly, the potent antioxidant $\mathrm{N}$-acetylcysteine showed a significant $40 \%$ decrease in the combined primary endpoint of cardiovascular events in another study [109]. After these rewarding results, we proposed the use of vitamin E coated dialysis membrane in these patients, which effectively reduces oxidative stress markers $[132,133]$. Finally, in another highrisk population of diabetics carrying the haptoglobin 2-2 genotype, which is associated with inferior antioxidant protection [134], vitamin E was able to reduce the primary composite end point of cardiovascular death, nonfatal myocardial infarction, or stroke [110], even on top of statin therapy [135].

Thus, most controlled randomized trials involving the use of antioxidants provided negative results. However, 
TABLE 2: Cohort studies demonstrating no association between oxidized low-density lipoprotein measurement and cardiovascular events.

\begin{tabular}{|c|c|c|c|c|c|c|}
\hline Oxidative oxLDL test & $\begin{array}{l}\text { Population } \\
\text { under study }\end{array}$ & CV endpoints & $\begin{array}{l}\text { Number of } \\
\text { events }\end{array}$ & Followup & Findings & Reference \\
\hline OxLDL Abs 4E06 & 3033 elderly & CV events & $418 \mathrm{IHD}, 120 \mathrm{MI}$ & 3 years & $\begin{array}{l}\text { OxLDL did not predict CV events } \\
\text { at MV analysis }\end{array}$ & $\begin{array}{l}\text { Holvoet et al. } \\
{[95]}\end{array}$ \\
\hline $\begin{array}{l}\text { AutoAbs } \\
\text { MDA-oxLDL }\end{array}$ & 2619 subjects & $\begin{array}{l}\text { IHD (angina, } \\
\text { ACS, and IHD } \\
\text { death); CV } \\
\text { events (IHD + } \\
\text { TIA/stroke) }\end{array}$ & $\begin{array}{c}151 \mathrm{IHD}, 234 \mathrm{CV} \\
\text { events }\end{array}$ & 8 years & $\begin{array}{c}\text { AutoAbs did not predict CV } \\
\text { events }\end{array}$ & $\begin{array}{l}\text { Wilson et al. } \\
\text { [99] }\end{array}$ \\
\hline $\begin{array}{l}\text { OxLDL Abs 4E06, } \\
\text { AutoAbs } \\
\text { MDA-/Cu-oxLDL }\end{array}$ & 919 subjects & $\begin{array}{c}\text { Carotid } \\
\text { atherosclerosis } \\
\text { progression }\end{array}$ & na & 5 years & $\begin{array}{l}\text { AutoAbs and oxLDL did not } \\
\text { predict CV events }\end{array}$ & $\begin{array}{l}\text { Mayr et al. } \\
\quad[100]\end{array}$ \\
\hline AutoAbs Cu-oxLDL & $\begin{array}{l}92 \text { NIDDM, } \\
80 \mathrm{ctr}\end{array}$ & CV events & $\begin{array}{l}34 \mathrm{CV} \text { events, } \\
15 \mathrm{CV} \text { deaths }\end{array}$ & 10 years & $\begin{array}{c}\text { AutoAbs did not predict CV } \\
\text { events }\end{array}$ & $\begin{array}{l}\text { Uusitupa et al. } \\
\text { [96] }\end{array}$ \\
\hline OxLDL Abs 4E06 & $\begin{array}{c}69 \text { ESRD on } \\
\text { hemodialysis, } \\
33 \mathrm{ctr}\end{array}$ & $\mathrm{CV}$ events & $18 \mathrm{CV}$ events & 43 months & OxLDL did not predict CV events & Lee et al. [101] \\
\hline AutoAbs Cu-oxLDL & $415 \mathrm{IHD}$ & CV events & $\begin{array}{c}35 \mathrm{CV} \\
\text { deaths/MI, } \\
\text { 33 PTCA/CABG }\end{array}$ & 5 years & $\begin{array}{c}\text { AutoAbs did not predict CV } \\
\text { events }\end{array}$ & $\begin{array}{l}\text { Erkkilä et al. } \\
\qquad \text { [97] }\end{array}$ \\
\hline OxLDL Abs 4E06 & 687 PTCA pts & $\begin{array}{c}\text { Restenosis, CV } \\
\text { events }\end{array}$ & $\begin{array}{l}135 \text { restenosis, } \\
181 \mathrm{CV} \text { events } \\
\end{array}$ & 1 year & OxLDL did not predict CV events & $\begin{array}{c}\text { Braun et al. } \\
{[98]}\end{array}$ \\
\hline
\end{tabular}

Abs: antibodies; AutoAbs: autoantibodies; CABG: coronary artery by-pass surgery; CHF: congestive heart failure; Crt: controls; CV: cardiovascular; ESRD: endstage renal disease; IHD: ischemic heart disease; IMT: intima-media thickness; MI: myocardial infarction; NIDDM: noninsulin dependent diabetes mellitus; oxLDL: oxidized low-density lipoproteins; PTCA: percutaneous transluminal coronary angioplasty; pts: patients.

TABLE 3: Randomized controlled trials demonstrating a beneficial effect of antioxidant therapy.

\begin{tabular}{|c|c|c|c|c|c|c|c|c|}
\hline Source & Patients & Inclusion criteria & $\begin{array}{l}\text { Antioxidant } \\
\text { agent }\end{array}$ & Dose & Route & Endpoints & Followup & Events \\
\hline CHAOS [106] & 2002 & $\begin{array}{c}\text { Angiographically } \\
\text { demonstrated } \\
\text { CAD }\end{array}$ & Vit E & $400 / 800 \mathrm{IU}$ & $\mathrm{PO}$ & $\begin{array}{l}\text { CV death + } \\
\text { MI; nonfatal } \\
\text { MI }\end{array}$ & $510 d$ & $\begin{array}{c}\text { CV death: } 27 \text { vit } \\
\text { E, } 23 \text { pl; nonfatal } \\
\text { MI: } 14 \text { vit E, } \\
41 \mathrm{pl}\end{array}$ \\
\hline WHS [107] & 39876 & Healthy women & Vit E & $\begin{array}{l}600 \mathrm{IU} \\
\mathrm{q} 48 \mathrm{~h}\end{array}$ & $\mathrm{PO}$ & $\begin{array}{c}\text { Composite } \\
\text { endpoint (CV } \\
\text { death, MI, and } \\
\text { stroke) }\end{array}$ & $10.1 \mathrm{y}$ & $\begin{array}{c}\text { CV events: Vit E } \\
\text { 482, pl 517; CV } \\
\text { death: Vit E 106, } \\
\text { pl 140; MI: Vit E } \\
\text { 196, pl } 195\end{array}$ \\
\hline SPACE [108] & 196 & $\begin{array}{c}\text { Hemodialysis CV } \\
\text { disease pts }\end{array}$ & Vit E & $800 \mathrm{IU}$ & $\mathrm{PO}$ & $\begin{array}{l}\text { Composite } \\
\text { endpoint (MI, } \\
\text { ACS, PAD, and } \\
\text { stroke) }\end{array}$ & $519 d$ & $\begin{array}{c}\text { Composite } \\
\text { endpoint: Vit E } \\
\text { 15, pl 33; } \\
\text { CV death: vit E } \\
\text { 9, pl 15; nonfatal } \\
\text { MI: vit E 8, pl } 18\end{array}$ \\
\hline $\begin{array}{l}\text { Tepel et al. } \\
{[109]}\end{array}$ & 134 & $\begin{array}{c}\text { Hemodialysis CV } \\
\text { disease pts }\end{array}$ & Acetylcysteine & $1200 \mathrm{mg}$ & $\mathrm{PO}$ & $\begin{array}{c}\text { Composite } \\
\text { endpoint (CV } \\
\text { death, MI, } \\
\text { PTCA/CABG, } \\
\text { PAD, and } \\
\text { stroke) }\end{array}$ & $14.5 \mathrm{~m}$ & $\begin{array}{l}\text { Composite } \\
\text { endpoint: } \\
\text { acetylcysteine } \\
18, \text { pl } 33\end{array}$ \\
\hline $\begin{array}{l}\text { Milman et al. } \\
\text { [110] }\end{array}$ & 1434 & $\begin{array}{l}\text { Diabetes mellitus } \\
\text { Hp 2-2 genotype }\end{array}$ & Vit E & $400 \mathrm{IU}$ & $\mathrm{PO}$ & $\begin{array}{c}\text { Composite } \\
\text { endpoint }(\mathrm{CV} \\
\text { death, MI, and } \\
\text { stroke) }\end{array}$ & $18 \mathrm{~m}$ & $\begin{array}{c}\text { Composite } \\
\text { endpoint: Vit E } \\
\text { 16, pl } 33\end{array}$ \\
\hline
\end{tabular}

CAD: coronary artery disease; CV: cardiovascular; d: days; DM: diabetes mellitus; HR: hazard ratio; HTN: arterial hypertension; m: months; MI: myocardial infarction; MLD: minimal luminal diameter; na: not available; PAD: peripheral artery disease, pl: placebo; PO: per os; pts: patients; RF: risk factor; vit: vitamin; y: years. 
TABLE 4: Randomized controlled trials demonstrating no effect of antioxidant therapy.

\begin{tabular}{|c|c|c|c|c|c|c|c|c|}
\hline Source & $\begin{array}{l}\text { Number } \\
\text { of patients }\end{array}$ & $\begin{array}{c}\text { Inclusion } \\
\text { criteria }\end{array}$ & $\begin{array}{l}\text { Antioxidant } \\
\text { agent }\end{array}$ & Dose & Route & Endpoints & Followup & Events \\
\hline $\begin{array}{l}\text { Virtamo et } \\
\text { al. [111] }\end{array}$ & 27271 & Male smokers & $\begin{array}{c}\text { Vit E, } \\
\text { beta-carotene }\end{array}$ & $\begin{array}{l}50 \mathrm{mg} \\
20 \mathrm{mg}\end{array}$ & $\mathrm{PO}$ & $\begin{array}{l}\text { Major } \\
\text { coronary } \\
\text { events }(\mathrm{CV} \\
\text { death, } \mathrm{MI})\end{array}$ & $6.1 \mathrm{y}$ & $\begin{array}{c}\text { CV events: Vit E 519, } \\
\text { beta-carotene 547, Vit E + } \\
\text { beta-carotene 511, and pl 534; CV } \\
\text { death: Vit E 212, beta-carotene } \\
\text { 235, Vit E + beta-carotene 222, } \\
\text { and pl 238; non-fatal MI: Vit E } \\
\text { 307, beta-carotene 312, Vit E + } \\
\text { beta-carotene 289, and pl } 296\end{array}$ \\
\hline $\begin{array}{l}\text { Rapola et al. } \\
{[112]}\end{array}$ & 1862 & Previous MI & $\begin{array}{c}\text { Vit E, } \\
\text { beta-carotene }\end{array}$ & $\begin{array}{l}50 \mathrm{mg} \\
20 \mathrm{mg}\end{array}$ & $\mathrm{PO}$ & $\begin{array}{c}\text { Major } \\
\text { coronary } \\
\text { events }(\mathrm{CV} \\
\text { death, MI) }\end{array}$ & $5.3 \mathrm{y}$ & $\begin{array}{c}\text { CV events: Vit E 94, } \\
\text { beta-carotene 113, Vit E + } \\
\text { beta-carotene 123, and pl } 94\end{array}$ \\
\hline HATS [113] & 80 & CAD & $\begin{array}{l}\text { Vit } \mathrm{E} / \mathrm{C} \text {, beta- } \\
\text { carotene, and } \\
\text { selenium }\end{array}$ & $\begin{array}{l}800 \mathrm{IU} \\
1 \mathrm{~g} \\
25 \mathrm{mg}, \\
\text { and } 100 \mathrm{~g}\end{array}$ & $\mathrm{PO}$ & $\begin{array}{l}\text { Composite } \\
\text { endpoint (CV } \\
\text { death, } \mathrm{MI} \text {, and } \\
\text { PTCA/CABG) }\end{array}$ & $38 \mathrm{~m}$ & $\begin{array}{c}\text { CV events: antiox 9, pl 9; CV } \\
\text { death: antiox } 0, \mathrm{pl} 1 \text {; nonfatal MI: } \\
\text { antiox } 1, \mathrm{pl} 4\end{array}$ \\
\hline PHS II [114] & 14641 & Male physicians & Vit E/C & $\begin{array}{l}400 \mathrm{IU} \\
500 \mathrm{mg}\end{array}$ & $\mathrm{PO}$ & $\begin{array}{c}\text { Composite } \\
\text { endpoint (CV } \\
\text { death, MI, and } \\
\text { stroke) }\end{array}$ & $8 y$ & $\begin{array}{l}\text { CV events: Vit E 620, pl 625; Vit } \\
\text { C 619, pl 626; CV death: Vit E } \\
\text { 258, pl 251; Vit C 256, pl 253; MI: } \\
\text { Vit E 240, pl 271; Vit C 260, pl } 251 \\
\end{array}$ \\
\hline WACS [115] & 8171 & $\begin{array}{l}\text { High CV risk } \\
\text { women }\end{array}$ & $\begin{array}{c}\text { Vit E/C, } \\
\text { beta-carotene }\end{array}$ & $\begin{array}{l}600 \mathrm{IU} \\
\mathrm{q} 48 \mathrm{~h}, \\
500 \mathrm{mg} \\
\text { and } \\
50 \mathrm{mg} \\
\mathrm{q} 48 \mathrm{~h}\end{array}$ & $\mathrm{PO}$ & $\begin{array}{l}\text { Composite } \\
\text { endpoint (CV } \\
\text { death, MI, } \\
\text { PTCA/CABG, } \\
\text { and stroke) }\end{array}$ & $9.4 \mathrm{y}$ & $\begin{array}{l}\text { CV events: Vit E 708, pl 742; Vit } \\
\text { C 731, pl 719; beta-carotene 731, } \\
\text { pl 719; CV death: Vit E 193, pl } \\
\text { 202; Vit C 206, pl 189; } \\
\text { beta-carotene 211, pl 184; MI: Vit } \\
\text { E 131, pl 143; Vit C 140, pl 134; } \\
\text { beta-carotene 135, pl 139 }\end{array}$ \\
\hline PPP [116] & 4495 & Subjects $\geq 1 \mathrm{RF}$ & Vit E & $300 \mathrm{mg}$ & $\mathrm{PO}$ & $\begin{array}{l}\text { Composite } \\
\text { endpoint (CV } \\
\text { death, MI, and } \\
\text { stroke) }\end{array}$ & $3.6 \mathrm{y}$ & $\begin{array}{l}\text { CV events: Vit E 56, pl 53; CV } \\
\text { death: Vit E 22, pl 26; MI: Vit E } \\
\text { 22, pl } 25\end{array}$ \\
\hline $\begin{array}{l}\text { GISSI- } \\
\text { prevenzione } \\
{[117]}\end{array}$ & 5660 & Recent MI & Vit E & $300 \mathrm{mg}$ & $\mathrm{PO}$ & $\begin{array}{c}\text { Composite } \\
\text { endpoint (CV } \\
\text { death, MI, and } \\
\text { stroke) } \\
\end{array}$ & $3.5 \mathrm{y}$ & $\begin{array}{l}\text { CV events: Vit E 371, pl 414; CV } \\
\text { death: Vit E 155, pl 193; MI: Vit E } \\
\text { 22, pl } 25\end{array}$ \\
\hline $\begin{array}{l}\text { Greenberg } \\
\text { et al. [118] }\end{array}$ & 1720 & Skin cancer & beta-carotene & $50 \mathrm{mg}$ & $\mathrm{PO}$ & CV death & $4.3 \mathrm{y}$ & CV death: beta-carotene 68, pl 59 \\
\hline PHS [119] & 22071 & Male physicians & beta-carotene & $\begin{array}{c}50 \mathrm{mg} \\
\mathrm{q} 48\end{array}$ & $\mathrm{PO}$ & $\begin{array}{l}\text { Malignant } \\
\text { neoplasm; } \\
\text { composite } \\
\text { endpoint }(\mathrm{CV} \\
\text { death, MI, and } \\
\text { stroke) } \\
\end{array}$ & $12 y$ & $\begin{array}{l}\text { CV events: beta-carotene } 967, \mathrm{pl} \\
\text { 972; CV death: beta-carotene } \\
\text { 338, pl 313; MI: beta-carotene } \\
\text { 468, pl } 489\end{array}$ \\
\hline $\begin{array}{l}\text { SUVIMAX } \\
{[120]}\end{array}$ & 13017 & Adult subjects & $\begin{array}{l}\text { Vit E/C, beta- } \\
\text { carotene, } \\
\text { selenium, } \\
\text { and zinc }\end{array}$ & $\begin{array}{l}30 \mathrm{mg} \\
120 \mathrm{mg}, \\
6 \mathrm{mg} \\
100 \mathrm{~g} \\
\text { and } \\
20 \mathrm{mg}\end{array}$ & $\mathrm{PO}$ & $\begin{array}{l}\mathrm{CV} \text { ischemic } \\
\text { events }\end{array}$ & $7.5 y$ & CV events: antiox 134, pl 137 \\
\hline HPS [121] & 20536 & $\begin{array}{c}\text { CAD, PAD, } \\
\mathrm{DM} \text {, and HTN }\end{array}$ & $\begin{array}{c}\text { Vit E/C, } \\
\text { beta-carotene }\end{array}$ & $\begin{array}{l}600 \mathrm{mg} \\
250 \mathrm{mg} \\
20 \mathrm{mg}\end{array}$ & $\mathrm{PO}$ & $\begin{array}{c}\text { Composite } \\
\text { endpoint }(\mathrm{CV} \\
\text { death, and } \mathrm{MI}) \\
\end{array}$ & $5 y$ & $\begin{array}{l}\text { CV death: antiox 878, pl 840; MI: } \\
\text { antiox 1063, pl 1047; CV events: } \\
\text { antiox 2306, pl } 2312\end{array}$ \\
\hline HOPE [122] & 9541 & $\begin{array}{l}\text { CV disease or } \\
\mathrm{DM}+ \\
\text { additional CV } \\
\mathrm{RF} \\
\end{array}$ & Vit E & $400 \mathrm{IU}$ & $\mathrm{PO}$ & $\begin{array}{l}\text { Composite } \\
\text { endpoint (CV } \\
\text { death, MI, and } \\
\text { stroke) }\end{array}$ & $7 y$ & $\begin{array}{l}\text { CV events: Vit E 1022, pl 985; CV } \\
\text { death: Vit E 482, pl 475; MI: Vit E } \\
\text { 724, pl } 686\end{array}$ \\
\hline
\end{tabular}


TABLE 4: Continued.

\begin{tabular}{|c|c|c|c|c|c|c|c|c|}
\hline Source & $\begin{array}{l}\text { Number } \\
\text { of patients }\end{array}$ & $\begin{array}{l}\text { Inclusion } \\
\text { criteria }\end{array}$ & $\begin{array}{c}\text { Antioxidant } \\
\text { agent }\end{array}$ & Dose & Route & Endpoints & Followup & Events \\
\hline $\begin{array}{l}\text { Mark et al. } \\
{[123]}\end{array}$ & 3318 & $\begin{array}{c}\text { Esophageal } \\
\text { dysplasia }\end{array}$ & $\begin{array}{c}\text { Vit E/C, } \\
\text { beta-carotene }\end{array}$ & $\begin{array}{l}60 \mathrm{IU} \\
180 \mathrm{mg} \\
\text { and } \\
15 \mathrm{mg}\end{array}$ & $\mathrm{PO}$ & CV death & $6 y$ & CV death: antiox 22, pl 35 \\
\hline $\begin{array}{l}\text { CARET } \\
{[124]}\end{array}$ & 1845 & $\begin{array}{l}\text { Exposure to } \\
\text { asbestos or } \\
\text { smoke }\end{array}$ & Vit E/A & $\begin{array}{l}15 / 30 \mathrm{mg}, \\
25000 \mathrm{IU}\end{array}$ & $\mathrm{PO}$ & $\begin{array}{l}\text { Lung cancer } \\
\text { incidence }\end{array}$ & $5.5 \mathrm{y}$ & CV death: HR 1.26 (0.99-1.61) \\
\hline WAVE [125] & 213 & $\begin{array}{l}\text { Postmenopausal } \\
\text { women with } \\
\text { CAD }\end{array}$ & Vit E/C & $\begin{array}{l}400 \mathrm{IU} \\
500 \mathrm{mg}\end{array}$ & $\mathrm{PO}$ & $\begin{array}{l}\text { Change in } \\
\text { MLD }\end{array}$ & $2.8 \mathrm{y}$ & $\begin{array}{l}\text { CV events: antiox 10, pl 5; CV } \\
\text { death: antiox 4, pl 2; nonfatal MI: } \\
\text { antiox 3, pl } 1\end{array}$ \\
\hline HOPE [126] & 9541 & $\begin{array}{l}\text { CV disease or } \\
\mathrm{DM}+ \\
\text { additional CV } \\
\mathrm{RF} \\
\end{array}$ & Vit E & $400 \mathrm{IU}$ & $\mathrm{PO}$ & $\begin{array}{l}\text { Composite } \\
\text { endpoint }(\mathrm{CV} \\
\text { death, } \mathrm{MI}, \\
\text { stroke) } \\
\end{array}$ & $4.5 \mathrm{y}$ & $\begin{array}{c}\text { CV events: Vit E 772, pl 739; CV } \\
\text { death: Vit E 342, pl 328; MI: Vit E } \\
\text { 532, pl } 524\end{array}$ \\
\hline
\end{tabular}

CAD: coronary artery disease; CV: cardiovascular; d: days; DM: diabetes mellitus; HR: hazard ratio; HTN: arterial hypertension; m: months; MI: myocardial infarction; MLD: minimal luminal diameter; na: not available; PAD: peripheral artery disease, pl: placebo; PO: per os; pts: patients; RF: risk factor; vit: vitamin; y: years.

administration of antioxidants to patients with known cardiovascular disease or with a very high-risk profile proved to be beneficial in a nontrivial number of studies.

\section{Conclusions}

Evidence supports on a molecular ground the oxidative hypothesis of atherosclerosis. The translation of experimental evidence in humans with studies aimed at the demonstration of the association of oxidative stress with cardiovascular events proved to be difficult and resulted in contrasting findings, particularly with administration of antioxidant therapy. However, the selection of patients either at higher risk or with cardiovascular disease provided much rewarding outcomes with numerous positive studies. It seems therefore that although this theory still needs further proofs to be definitely clarified, data available so far strengthen the pivotal role for oxidative stress in atherosclerosis.

\section{References}

[1] P. Libby, P. M. Ridker, and G. K. Hansson, "Progress and challenges in translating the biology of atherosclerosis," Nature, vol. 473, no. 7347, pp. 317-325, 2011.

[2] M. W. Majesky, "Developmental basis of vascular smooth muscle diversity," Arteriosclerosis, Thrombosis, and Vascular Biology, vol. 27, no. 6, pp. 1248-1258, 2007.

[3] M. A. Gimbrone Jr., J. N. Topper, T. Nagel, K. R. Anderson, and G. Garcia-Cardeña, "Endothelial dysfunction, hemodynamic forces, and atherogenesis," Annals of the New York Academy of Sciences, vol. 902, pp. 230-240, 2000.

[4] I. Tabas, K. J. Williams, and J. Borén, "Subendothelial lipoprotein retention as the initiating process in atherosclerosis: update and therapeutic implications," Circulation, vol. 116, no. 16, pp. 1832-1844, 2007.

[5] K. Skålén, M. Gustafsson, E. Knutsen Rydberg et al., "Subendothelial retention of atherogenic lipoproteins in early atherosclerosis," Nature, vol. 417, no. 6890, pp. 750-754, 2002.

[6] M. Navab, J. A. Berliner, A. D. Watson et al., "The Yin and Yang of oxidation in the development of the fatty streak: a review based on the 1994 George Lyman Duff memorial lecture," Arteriosclerosis, Thrombosis, and Vascular Biology, vol. 16, no. 7, pp. 831-842, 1996.

[7] J. A. Berliner, M. C. Territo, A. Sevanian et al., "Minimally modified low density lipoprotein stimulates monocyte endothelial interactions," Journal of Clinical Investigation, vol. 85, no. 4, pp. 1260-1266, 1990.

[8] S.-H. Choi, R. Harkewicz, J. H. Lee et al., "Lipoprotein accumulation in macrophages via toll-like receptor-4-dependent fluid phase uptake," Circulation Research, vol. 104, no. 12, pp. 13551363, 2009.

[9] Y. I. Miller, S. Viriyakosol, D. S. Worrall, A. Boullier, S. Butler, and J. L. Witztum, "Toll-like receptor 4-dependent and -independent cytokine secretion induced by minimally oxidized low-density lipoprotein in macrophages," Arteriosclerosis, Thrombosis, and Vascular Biology, vol. 25, no. 6, pp. 1213-1219, 2005.

[10] Y. S. Bae, J. H. Lee, S. H. Choi et al., "Macrophages generate reactive oxygen species in response to minimally oxidized lowdensity lipoprotein: toll-like receptor 4 - and spleen tyrosine kinase-dependent activation of NADPH oxidase 2," Circulation Research, vol. 104, no. 2, pp. 210-218, 2009.

[11] F. Parhami, Z. T. Fang, A. M. Fogelman, A. Andalibi, M. C. Territo, and J. A. Berliner, "Minimally modified low density lipoprotein-induced inflammatory responses in endothelial cells are mediated by cyclic adenosine monophosphate," Journal of Clinical Investigation, vol. 92, no. 1, pp. 471-478, 1993.

[12] T. Henriksen, E. M. Mahoney, and D. Steinberg, "Enhanced macrophage degradation of low density lipoprotein previously incubated with cultured endothelial cells: recognition by receptors for acetylated low density lipoproteins," Proceedings of the National Academy of Sciences of the United States of America, vol. 78, no. 10 I, pp. 6499-6503, 1981. 
[13] I. Tabas, "Macrophage death and defective inflammation resolution in atherosclerosis," Nature Reviews Immunology, vol. 10, no. 1, pp. 36-46, 2010.

[14] J. A. Ambrose, M. A. Tannenbaum, D. Alexopoulos et al., "Angiographic progression of coronary artery disease and the development of myocardial infarction," Journal of the American College of Cardiology, vol. 12, no. 1, pp. 56-62, 1988.

[15] W. C. Little, M. Constantinescu, R. J. Applegate et al., "Can coronary angiography predict the site of a subsequent myocardial infarction in patients with mild-to-moderate coronary artery disease?" Circulation, vol. 78, no. 5 I, pp. 1157-1166, 1988.

[16] R. Virmani, F. D. Kolodgie, A. P. Burke, A. Farb, and S. M. Schwartz, "Lessons from sudden coronary death: a comprehensive morphological classification scheme for atherosclerotic lesions," Arteriosclerosis, Thrombosis, and Vascular Biology, vol. 20, no. 5, pp. 1262-1275, 2000.

[17] G. W. Stone, A. Maehara, A. J. Lansky et al., "A prospective natural-history study of coronary atherosclerosis," New England Journal of Medicine, vol. 364, no. 3, pp. 226-235, 2011.

[18] P. Libby, "Molecular and cellular mechanisms of the thrombotic complications of atherosclerosis," Journal of Lipid Research, vol. 50, pp. S352-S357, 2009.

[19] D. Steinberg, S. Parthasarathy, T. E. Carew, J. C. Khoo, and J. L. Witztum, "Beyond cholesterol: modifications of low-density lipoprotein that increase its atherogenicity," New England Journal of Medicine, vol. 320, no. 14, pp. 915-924, 1989.

[20] U. P. Steinbrecher, S. Parthasarathy, and D. S. Leake, "Modification of low density lipoprotein by endothelial cells involves lipid peroxidation and degradation of low density lipoprotein phospholipids," Proceedings of the National Academy of Sciences of the United States of America, vol. 81, no. 12 I, pp. 3883-3887, 1984.

[21] T. Henriksen, E. M. Mahoney, and D. Steinberg, "Enhanced macrophage degradation of biologically modified low density lipoprotein," Arteriosclerosis, vol. 3, no. 2, pp. 149-159, 1983.

[22] J. W. Heinecke, H. Rosen, and A. Chait, "Iron and copper promote modification of low density lipoprotein by human arterial smooth muscle cells in culture," Journal of Clinical Investigation, vol. 74, no. 5, pp. 1890-1894, 1984.

[23] H. S. Kruth, N. L. Jones, W. Huang et al., "Macropinocytosis is the endocytic pathway that mediates macrophage foam cell formation with native low density lipoprotein," Journal of Biological Chemistry, vol. 280, no. 3, pp. 2352-2360, 2005.

[24] C. K. Glass and J. L. Witztum, "Atherosclerosis: the road ahead," Cell, vol. 104, no. 4, pp. 503-516, 2001.

[25] T. Cyrus, J. L. Witztum, D. J. Rader et al., "Disruption of the 12/15-lipoxygenase gene diminishes atherosclerosis in apo Edeficient mice," Journal of Clinical Investigation, vol. 103, no. 11, pp. 1597-1604, 1999.

[26] S. Yla-Herttuala, M. E. Rosenfeld, S. Parthasarathy et al., "Colocalization of 15-lipoxygenase mRNA and protein with epitopes of oxidized low density lipoprotein in macrophagerich areas of atherosclerotic lesions," Proceedings of the National Academy of Sciences of the United States of America, vol. 87, no. 18, pp. 6959-6963, 1990.

[27] S. M. Rankin, S. Parthasarathy, and D. Steinberg, "Evidence for a dominant role of lipoxygenase(s) in the oxidation of LDL by mouse peritoneal macrophages," Journal of Lipid Research, vol. 32, no. 3, pp. 449-456, 1991.

[28] S. M. Sendobry, J. A. Cornicelli, K. Welch et al., "Attenuation of diet-induced atherosclerosis in rabbits with a highly selective 15-lipoxygenase inhibitor lacking significant antioxidant properties," British Journal of Pharmacology, vol. 120, no. 7, pp. 11991206, 1997.

[29] T. M. A. Bocan, W. S. Rosebury, S. B. Mueller et al., "A specific 15-lipoxygenase inhibitor limits the progression and monocyte-macrophage enrichment of hypercholesterolemiainduced atherosclerosis in the rabbit," Atherosclerosis, vol. 136, pp. 203-216, 1998.

[30] A. Daugherty, J. L. Dunn, D. L. Rateri, and J. W. Heinecke, "Myeloperoxidase, a catalyst for lipoprotein oxidation, is expressed in human atherosclerotic lesions," Journal of Clinical Investigation, vol. 94, no. 1, pp. 437-444, 1994.

[31] E. A. Podrez, M. Febbraio, N. Sheibani et al., "Macrophage scavenger receptor CD36 is the major receptor for LDL modified by monocyte-generated reactive nitrogen species," Journal of Clinical Investigation, vol. 105, pp. 1095-1108, 2000.

[32] Z. Wang, S. J. Nicholls, E. R. Rodriguez et al., "Protein carbamylation links inflammation, smoking, uremia and atherogenesis," Nature Medicine, vol. 13, no. 10, pp. 1176-1184, 2007.

[33] J. L. Goldstein, Y. K. Ho, S. K. Basu, and M. S. Brown, "Binding site on macrophages that mediates uptake and degradation of acetylated low density lipoprotein, producing massive cholesterol deposition," Proceedings of the National Academy of Sciences of the United States of America, vol. 76, no. 1, pp. 333$337,1979$.

[34] M. T. Quinn, S. Parthasarathy, and D. Steinberg, "Lysophosphatidylcholine: a chemotactic factor for human monocytes and its potential role in atherogenesis," Proceedings of the National Academy of Sciences of the United States of America, vol. 85, no. 8, pp. 2805-2809, 1988.

[35] J. Frostegard, A. Haegerstrand, M. Gidlund, and J. Nilsson, "Biologically modified LDL increases the adhesive properties of endothelial cells," Atherosclerosis, vol. 90, no. 2-3, pp. 119-126, 1991.

[36] L. Cominacini, U. Garbin, A. F. Pasini et al., "Antioxidants inhibit the expression of intercellular cell adhesion molecule-1 and vascular cell adhesion molecule- 1 induced by oxidized LDL on human umbilical vein endothelial cells," Free Radical Biology and Medicine, vol. 22, no. 1-2, pp. 117-127, 1996.

[37] S. Yui, T. Sasaki, A. Miyazaki, S. Horiuchi, and M. Yamazaki, "Induction of murine macrophage growth by modified LDLs," Arteriosclerosis and Thrombosis, vol. 13, no. 3, pp. 331-337, 1993.

[38] M. T. Quinn, S. Parthasarathy, L. G. Fong, and D. Steinberg, "Oxidatively modified low density lipoproteins: a potential role in recruitment and retention of monocyte/macrophages during atherogenesis," Proceedings of the National Academy of Sciences of the United States of America, vol. 84, no. 9, pp. 2995-2998, 1987.

[39] A. Stiko-Rahm, A. Hultgardh-Nilsson, J. Regnstrom, A. Hamsten, and J. Nilsson, "Native and oxidized LDL enhances production of PDGF AA and the surface expression of PDGF receptors in cultured human smooth muscle cells," Arteriosclerosis and Thrombosis, vol. 12, no. 9, pp. 1099-1109, 1992.

[40] M. Kohno, K. Yokokawa, K. Yasunari et al., "Induction by lysophosphatidylcholine, a major phospholipid component of atherogenic lipoproteins, of human coronary artery smooth muscle cell migration," Circulation, vol. 98, no. 4, pp. 353-359, 1998.

[41] J. G. Kim, W. R. Taylor, and S. Parthasarathy, "Demonstration of the presence of lipid peroxide-modified proteins in human atherosclerotic lesions using a novel lipid peroxide-modified 
anti-peptide antibody," Atherosclerosis, vol. 143, no. 2, pp. 335340, 1999.

[42] V. Lindner, D. A. Lappi, A. Baird, R. A. Majack, and M. A. Reidy, "Role of basic fibroblast growth factor in vascular lesion formation," Circulation Research, vol. 68, no. 1, pp. 106-113, 1991.

[43] S. Jimi, K. Saku, N. Uesugi, N. Sakata, and S. Takebayashi, "Oxidized low density lipoprotein stimulates collagen production in cultured arterial smooth muscle cells," Atherosclerosis, vol. 116, no. 1, pp. 15-26, 1995.

[44] T. B. Rajavashisth, J. K. Liao, Z. S. Galis et al., "Inflammatory cytokines and oxidized low density lipoproteins increase endothelial cell expression of membrane type 1-matrix metalloproteinase," Journal of Biological Chemistry, vol. 274, no. 17, pp. 11924-11929, 1999.

[45] X.-P. Xu, S. R. Meisel, J. M. Ong et al., "Oxidized lowdensity lipoprotein regulates matrix metalloproteinase-9 and its tissue inhibitor in human monocyte-derived macrophages," Circulation, vol. 99, no. 8, pp. 993-998, 1999.

[46] A. Loidl, R. Claus, E. Ingolic, H.-P. Deigner, and A. Hermetter, "Role of ceramide in activation of stress-associated MAP kinases by minimally modified LDL in vascular smooth muscle cells," Biochimica et Biophysica Acta, vol. 1690, no. 2, pp.150-158, 2004.

[47] C. J. Schwartz, A. J. Valente, E. A. Sprague, J. L. Kelley, and R. M. Nerem, "The pathogenesis of atherosclerosis: an overview," Clinical Cardiology, vol. 14, no. 2, pp. 1-16, 1991.

[48] M. K. Cathcart, D. W. Morel, and G. M. Chisolm III, "Monocytes and neutrophils oxidize low density lipoprotein making it cytotoxic," Journal of Leukocyte Biology, vol. 38, no. 2, pp. 341350, 1985.

[49] M. Sata and K. Walsh, "Oxidized LDL activates Fas-mediated endothelial cell apoptosis," Journal of Clinical Investigation, vol. 102, no. 9, pp. 1682-1689, 1998.

[50] S. J. Hardwick, L. Hegyi, K. Clare et al., "Apoptosis in human monocyte-macrophages exposed to oxidized low density lipoprotein," Journal of Pathology, vol. 179, pp. 294-302, 1996.

[51] E. Thorin, C. A. Hamilton, M. H. Dominiczak, and J. L. Reid, "Chronic exposure of cultured bovine endothelial cells to oxidized LDL abolishes prostacyclin release," Arteriosclerosis and Thrombosis, vol. 14, no. 3, pp. 453-459, 1994.

[52] L.-X. Li, J.-X. Chen, D.-F. Liao, and L. Yu, "Probucol inhibits oxidized-low density lipoprotein-induced adhesion of monocytes to endothelial cells by reducing P-selectin synthesis in vitro," Endothelium, vol. 6, no. 1, pp. 1-8, 1998.

[53] D. A. Armstrong, "Oxidized LDL, ceroid, and prostaglandin metabolism in human atherosclerosis," Medical Hypotheses, vol. 38, no. 3, pp. 244-248, 1992.

[54] K. Kugiyama, T. Sakamoto, I. Misumi et al., “Transferable lipids in oxidized low-density lipoprotein stimulate plasminogen activator inhibitor-1 and inhibit tissue-type plasminogen activator release from endothelial cells," Circulation Research, vol. 73, no. 2, pp. 335-343, 1993.

[55] M. Gräfe, W. Auch-Schwelk, H. Hertel et al., "Human cardiac microvascular and macrovascular endothelial cells respond differently to oxidatively modified LDL," Atherosclerosis, vol.137, no. 1, pp. 87-95, 1998.

[56] B. A. Allison, L. Nilsson, F. Karpe, A. Hamsten, and P. Eriksson, "Effects of native, triglyceride-enriched, and oxidatively modified LDL on plasminogen activator inhibitor-1 expression in human endothelial cells," Arteriosclerosis, Thrombosis, and Vascular Biology, vol. 19, no. 5, pp. 1354-1360, 1999.
[57] J. K. Liao, W. S. S. Wee Soo Shin, W. Y. L. Wen Yee Lee, and S. L. Clark, "Oxidized low-density lipoprotein decreases the expression of endothelial nitric oxide synthase," Journal of Biological Chemistry, vol. 270, no. 1, pp. 319-324, 1995.

[58] C. M. Boulanger, F. C. Tanner, M.-L. Bea, A. W. A. Hahn, A. Werner, and T. F. Luscher, "Oxidized low density lipoproteins induce mRNA expression and release of endothelin from human and porcine endothelium," Circulation Research, vol. 70, no. 6, pp. 1191-1197, 1992.

[59] D. Harats, A. Shaish, J. George et al., "Overexpression of 15-lipoxygenase in vascular endothelium accelerates early atherosclerosis in LDL receptor-deficient mice," Arteriosclerosis, Thrombosis, and Vascular Biology, vol. 20, no. 9, pp. 2100-2105, 2000.

[60] J. Shen, E. Herderick, J. F. Cornhill et al., "Macrophagemediated 15-lipoxygenase expression protects against atherosclerosis development," Journal of Clinical Investigation, vol. 98, no. 10, pp. 2201-2208, 1996.

[61] T. Cyrus, D. Praticò, L. Zhao et al., "Absence of 12/15lipoxygenase expression decreases lipid peroxidation and atherogenesis in apolipoprotein e-deficient mice," Circulation, vol. 103, no. 18, pp. 2277-2282, 2001.

[62] J. George, A. Afek, A. Shaish et al., "12/15-Lipoxygenase gene disruption attenuates atherogenesis in LDL receptor-deficient mice," Circulation, vol. 104, no. 14, pp. 1646-1650, 2001.

[63] L. Zhao, C. A. Cuff, E. Moss et al., "Selective interleukin-12 synthesis defect in 12/15-lipoxygenase-deficient macrophages associated with reduced atherosclerosis in a mouse model of familial hypercholesterolemia," Journal of Biological Chemistry, vol. 277, no. 38, pp. 35350-35356, 2002.

[64] H. Huo, "Critical role of macrophage 12/15-lipoxygenase for atherosclerosis in apolipoprotein E-deficient mice," Circulation, vol. 110, pp. 2024-2031, 2004.

[65] A. J. Merched, K. Ko, K. H. Gotlinger, C. N. Serhan, and L. Chan, "Atherosclerosis: evidence for impairment of resolution of vascular inflammation governed by specific lipid mediators," FASEB Journal, vol. 22, no. 10, pp. 3595-3606, 2008.

[66] V. V. Kunjathoor, M. Febbraio, E. A. Podrez et al., "Scavenger receptors class A-I/II and CD36 are the principal receptors responsible for the uptake of modified low density lipoprotein leading to lipid loading in macrophages," Journal of Biological Chemistry, vol. 277, no. 51, pp. 49982-49988, 2002.

[67] M. Febbraio, E. A. Podrez, J. D. Smith et al., "Targeted disruption of the class $\mathrm{B}$, scavenger receptor $\mathrm{CD} 36$ protects against atherosclerotic lesion development in mice," Journal of Clinical Investigation, vol. 105, no. 8, pp. 1049-1056, 2000.

[68] H. Suzuki, Y. Kurihara, M. Takeya et al., "A role for macrophage scavenger receptors in atherosclerosis and susceptibility to infection," Nature, vol. 386, no. 6622, pp. 292-296, 1997.

[69] K. J. Moore, V. V. Kunjathoor, S. L. Koehn et al., "Loss of receptor-mediated lipid uptake via scavenger receptor A or CD36 pathways does not ameliorate atherosclerosis in hyperlipidemic mice," Journal of Clinical Investigation, vol. 115, no. 8, pp. 2192-2201, 2005.

[70] J. L. Witztum, “You are right too!” Journal of Clinical Investigation, vol. 115, no. 8, pp. 2072-2075, 2005.

[71] J. L. Witztum and D. Steinberg, "The oxidative modification hypothesis of atherosclerosis: does it hold for humans?" Trends in Cardiovascular Medicine, vol. 11, no. 3-4, pp. 93-102, 2001.

[72] D. Praticò, R. K. Tangirala, D. J. Rader, J. Rokach, and G. A. Fitzgerald, "Vitamin E suppresses isoprostane generation 
in vivo and reduces atherosclerosis in ApoE-deficient mice," Nature Medicine, vol. 4, no. 10, pp. 1189-1192, 1998.

[73] A. T. Erkkilä, O. Närvänen, S. Lehto, M. I. J. Uusitupa, and S. Ylä-Herttuala, "Autoantibodies against oxidized low-density lipoprotein and cardiolipin in patients with coronary heart disease," Arteriosclerosis, Thrombosis, and Vascular Biology, vol. 20, no. 1, pp. 204-209, 2000.

[74] T. Inoue, T. Uchida, H. Kamishirado, K. Takayanagi, T. Hayashi, and S. Morooka, "Clinical significance of antibody against oxidized low density lipoprotein in patients with atherosclerotic coronary artery disease," Journal of the American College of Cardiology, vol. 37, no. 3, pp. 775-779, 2001.

[75] S. Tsimikas, "Measures of oxidative stress," Clinics in Laboratory Medicine, vol. 26, no. 3, pp. 571-590, 2006.

[76] N. A. Strobel, R. G. Fassett, S. A. Marsh, and J. S. Coombes, "Oxidative stress biomarkers as predictors of cardiovascular disease," International Journal of Cardiology, vol. 147, no. 2, pp. 191-201, 2011.

[77] K. Nishi, H. Itabe, M. Uno et al., "Oxidized LDL in carotid plaques and plasma associates with plaque instability," Arteriosclerosis, Thrombosis, and Vascular Biology, vol. 22, no. 10, pp. 1649-1654, 2002.

[78] S. Tsimikas, E. S. Brilakis, E. R. Miller et al., "Oxidized phospholipids, Lp(a) lipoprotein, and coronary artery disease," New England Journal of Medicine, vol. 353, no. 1, pp. 46-57, 2005.

[79] G. P. Rossi, M. Cesari, R. De Toni et al., "Antibodies to oxidized low-density lipoproteins and angiographically assessed coronary artery disease in white patients," Circulation, vol. 108, no. 20, pp. 2467-2472, 2003.

[80] S. Tsimikas, E. S. Brilakis, R. J. Lennon et al., "Relationship of IgG and IgM autoantibodies to oxidized low density lipoprotein with coronary artery disease and cardiovascular events," Journal of Lipid Research, vol. 48, no. 2, pp. 425-433, 2007.

[81] G. Maiolino, L. Pedon, M. Cesari et al., "Antibodies to malondialdehyde oxidized low-density lipoproteins predict long term cardiovascular mortality in high risk patients," International Journal of Cardiology, 2013.

[82] B. Röhrig, J.-B. Du Prel, D. Wachtlin, and M. Blettner, “Types of study in medical research-part 3 of a series on evaluation of scientific publications," Deutsches Arzteblatt, vol. 106, no. 15, pp. 262-268, 2009.

[83] T. Tsutsui, T. Tsutamoto, A. Wada et al., "Plasma oxidized low-density lipoprotein as a prognostic predictor in patients with chronic congestive heart failure," Journal of the American College of Cardiology, vol. 39, no. 6, pp. 957-962, 2002.

[84] T. Shoji, M. Fukumoto, E. Kimoto et al., "Antibody to oxidized low-density lipoprotein and cardiovascular mortality in endstage renal disease," Kidney International, vol. 62, no. 6, pp. 2230-2237, 2002.

[85] B. Bayés, M. Cruz Pastor, J. Bonal et al., "Homocysteine, Creactive protein, lipid peroxidation and mortality in haemodialysis patients," Nephrology Dialysis Transplantation, vol. 18, no. 1, pp. 106-112, 2003.

[86] B. Bayés, M. C. Pastor, J. Bonal, A. Foraster, and R. Romero, "Oxidative stress, inflammation and cardiovascular mortality in haemodialysis-role of seniority and intravenous ferrotherapy: analysis at 4 years of follow-up," Nephrology Dialysis Transplantation, vol. 21, no. 4, pp. 984-990, 2006.

[87] K. Shimada, H. Mokuno, E. Matsunaga et al., "Circulating oxidized low-density lipoprotein is an independent predictor for cardiac event in patients with coronary artery disease," Atherosclerosis, vol. 174, no. 2, pp. 343-347, 2004.
[88] K. Wallenfeldt, B. Fagerberg, J. Wikstrand, and J. Hulthe, "Oxidized low-density lipoprotein in plasma is a prognostic marker of subclinical atherosclerosis development in clinically healthy men," Journal of Internal Medicine, vol. 256, no. 5, pp. 413-420, 2004.

[89] T. Naruko, M. Ueda, S. Ehara et al., "Persistent high levels of plasma oxidized low-density lipoprotein after acute myocardial infarction predict stent restenosis," Arteriosclerosis, Thrombosis, and Vascular Biology, vol. 26, no. 4, pp. 877-883, 2006.

[90] S. Tsimikas, S. Kiechl, J. Willeit et al., "Oxidized phospholipids predict the presence and progression of carotid and femoral atherosclerosis and symptomatic cardiovascular disease: fiveyear prospective results from the bruneck study," Journal of the American College of Cardiology, vol. 47, no. 11, pp. 2219-2228, 2006.

[91] N. Johnston, T. Jernberg, B. Lagerqvist, A. Siegbahn, and L. Wallentin, "Oxidized low-density lipoprotein as a predictor of outcome in patients with unstable coronary artery disease," International Journal of Cardiology, vol. 113, no. 2, pp. 167-173, 2006.

[92] S. Kiechl, J. Willeit, M. Mayr et al., "Oxidized phospholipids, lipoprotein(a), lipoprotein-associated phospholipase A2 Activity, and 10-year cardiovascular outcomes: prospective results from the bruneck study," Arteriosclerosis, Thrombosis, and Vascular Biology, vol. 27, no. 8, pp. 1788-1795, 2007.

[93] M. F. Lopes-Virella, K. J. Hunt, N. L. Baker et al., “The levels of MDA-LDL in circulating immune complexes predict myocardial infarction in the VADT study," Atherosclerosis, vol. 224, pp. 526-531, 2012.

[94] S. Tsimikas, P. Willeit, J. Willeit et al., "Oxidation-specific biomarkers, prospective 15-year cardiovascular and stroke outcomes, and net reclassification of cardiovascular events," Journal of the American College of Cardiology, vol. 60, pp. 2218-2229, 2012.

[95] P. Holvoet, S. B. Kritchevsky, R. P. Tracy et al., "The metabolic syndrome, circulating oxidized LDL, and risk of myocardial infarction in well-functioning elderly people in the health, aging, and body composition cohort," Diabetes, vol. 53, no. 4, pp. 1068-1073, 2004.

[96] M. I. J. Uusitupa, L. Niskanen, J. Luoma et al., "Autoantibodies against oxidized LDL do not predict atherosclerotic vascular disease in non-insulin-dependent diabetes mellitus," Arteriosclerosis, Thrombosis, and Vascular Biology, vol. 16, no. 10, pp. 1236-1242, 1996.

[97] A. T. Erkkilä, O. Närvänen, S. Lehto, M. I. J. Uusitupa, and S. Ylä-Herttuala, "Antibodies against oxidized LDL and cardiolipin and mortality in patients with coronary heart disease," Atherosclerosis, vol. 183, no. 1, pp. 157-162, 2005.

[98] S. Braun, G. Ndrepepa, N. Von Beckerath et al., "Lack of association between circulating levels of plasma oxidized lowdensity lipoproteins and clinical outcome after coronary stenting," American Heart Journal, vol. 150, no. 3, pp. 550-556, 2005.

[99] P. W. F. Wilson, O. Ben-Yehuda, J. McNamara, J. Massaro, J. Witztum, and P. D. Reaven, "Autoantibodies to oxidized LDL and cardiovascular risk. The Framingham Offspring Study," Atherosclerosis, vol. 189, no. 2, pp. 364-368, 2006.

[100] M. Mayr, S. Kiechl, S. Tsimikas et al., "Oxidized low-density lipoprotein autoantibodies, chronic infections, and carotid atherosclerosis in a population-based study," Journal of the American College of Cardiology, vol. 47, no. 12, pp. 2436-2443, 2006. 
[101] Y. K. Lee, D. H. Lee, J. K. Kim et al., "Lysophosphatidylcholine, oxidized low-density lipoprotein and cardiovascular disease in korean hemodialysis patients: analysis at 5 years of follow-up," Journal of Korean Medical Science, vol. 28, pp. 268-273, 2013.

[102] J. P. T. Higgins and S. Green, Cochrane Handbook for Systematic Reviews of Interventions, version 5.1.0, The Cochrane Collaboration, 2011.

[103] D. A. Grimes and K. F. Schulz, "Bias and causal associations in observational research," The Lancet, vol. 359, no. 9302, pp. 248252, 2002.

[104] P. A. Rochon, J. H. Gurwitz, K. Sykora et al., "Reader's guide to critical appraisal of cohort studies-1. Role and design," British Medical Journal, vol. 330, no. 7496, pp. 895-897, 2005.

[105] J. George, D. Harats, E. Bakshi et al., "Anti-oxidized low density lipoprotein antibody determination as a predictor of restenosis following percutaneous transluminal coronary angioplasty," Immunology Letters, vol. 68, no. 2-3, pp. 263-266, 1999.

[106] N. G. Stephens, A. Parsons, P. M. Schofield et al., "Randomised controlled trial of vitamin $\mathrm{E}$ in patients with coronary disease: Cambridge Heart Antioxidant Study (CHAOS)," The Lancet, vol. 347, no. 9004, pp. 781-786, 1996.

[107] I.-M. Lee, N. R. Cook, J. M. Gaziano et al., "Vitamin E in the primary prevention of cardiovascular disease and cancer. The women's health study: a randomized controlled trial," Journal of the American Medical Association, vol. 294, no. 1, pp. 56-65, 2005.

[108] M. Boaz, S. Smetana, T. Weinstein et al., "Secondary prevention with antioxidants of cardiovascular disease in endstage renal disease (SPACE): randomised placebo-controlled trial," The Lancet, vol. 356, no. 9237, pp. 1213-1218, 2000.

[109] M. Tepel, M. Van der Giet, M. Statz, J. Jankowski, and W. Zidek, "The antioxidant acetylcysteine reduces cardiovascular events in patients with end-stage renal failure: a randomized, controlled trial," Circulation, vol. 107, no. 7, pp. 992-995, 2003.

[110] U. Milman, S. Blum, C. Shapira et al., "Vitamin E supplementation reduces cardiovascular events in a subgroup of middleaged individuals with both type 2 diabetes mellitus and the haptoglobin 2-2 genotype: a prospective double-blinded clinical trial," Arteriosclerosis, Thrombosis, and Vascular Biology, vol. 28, no. 2, pp. 341-347, 2008.

[111] J. Virtamo, J. M. Rapola, S. Ripatti et al., "Effect of vitamin E and beta carotene on the incidence of primary nonfatal myocardial infarction and fatal coronary heart disease," Archives of Internal Medicine, vol. 158, no. 6, pp. 668-675, 1998.

[112] J. M. Rapola, J. Virtamo, S. Ripatti et al., "Randomised trial of $\alpha$ tocopherol and $\beta$-carotene supplements on incidence of major coronary events in men with previous myocardial infarction," The Lancet, vol. 349, no. 9067, pp. 1715-1720, 1997.

[113] B. G. Brown, X.-Q. Zhao, A. Chait et al., "Simvastatin and niacin, antioxidant vitamins, or the combination for the prevention of coronary disease," New England Journal of Medicine, vol. 345, no. 22, pp. 1583-1592, 2001.

[114] H. D. Sesso, J. E. Buring, W. G. Christen et al., "Vitamins E and $\mathrm{C}$ in the prevention of cardiovascular disease in men: the physicians' health study II randomized controlled trial," Journal of the American Medical Association, vol. 300, no. 18, pp. 21232133, 2008.

[115] N. R. Cook, C. M. Albert, J. M. Gaziano et al., "A randomized factorial trial of vitamins $\mathrm{C}$ and $\mathrm{E}$ and beta carotene in the secondary prevention of cardiovascular events in women: results from the women's antioxidant cardiovascular study," Archives of Internal Medicine, vol. 167, no. 15, pp. 1610-1618, 2007.
[116] G. de Gaetano and Collaborative Group of the Primary Prevention Project, "Low-dose aspirin and vitamin E in people at cardiovascular risk: a randomised trial in general practice. collaborative group of the primary prevention project," The Lancet, vol. 357, pp. 89-95, 2001.

[117] GISSI-Prevenzione Investigators, "Dietary supplementation with n-3 polyunsaturated fatty acids and vitamin $\mathrm{E}$ after myocardial infarction: results of the GISSI-prevenzione trial. gruppo italiano per lo studio della sopravvivenza nell'infarto miocardico," The Lancet, vol. 354, pp. 447-455, 1999.

[118] E. R. Greenberg, J. A. Baron, M. R. Karagas et al., "Mortality associated with low plasma concentration of beta carotene and the effect of oral supplementation," Journal of the American Medical Association, vol. 275, no. 9, pp. 699-703, 1996.

[119] C. H. Hennekens, J. E. Buring, J. E. Manson et al., "Lack of effect of long-term supplementation with beta carotene on the incidence of malignant neoplasms and cardiovascular disease," New England Journal of Medicine, vol. 334, no. 18, pp. 1145-1149, 1996.

[120] S. Hercberg, P. Galan, P. Preziosi et al., "The SU.VI.MAX study: a randomized, placebo-controlled trial of the health effects of antioxidant vitamins and minerals," Archives of Internal Medicine, vol. 164, no. 21, pp. 2335-2342, 2004.

[121] R. Collins, J. Armitage, S. Parish, P. Sleight, and R. Peto, "MRC/BHF Heart Protection Study of antioxidant vitamin supplementation in 20536 high-risk individuals: a randomised placebo-controlled trial," The Lancet, vol. 360, no. 9326, pp. 2333, 2002.

[122] E. Lonn, "Effects of long-term vitamin E supplementation on cardiovascular events and cancer: a randomized controlled trial," Journal of the American Medical Association, vol. 293, no. 11, pp. 1338-1347, 2005.

[123] S. D. Mark, W. Wang, J. F. Fraumeni Jr. et al., "Lowered risks of hypertension and cerebrovascular disease after vitamin/mineral supplementation: the Linxian nutrition intervention trial," American Journal of Epidemiology, vol. 143, no. 7, pp. 658-664, 1996.

[124] G. S. Omenn, G. E. Goodman, M. D. Thornquist et al., "Effects of a combination of beta carotene and vitamin A on lung cancer and cardiovascular disease," New England Journal of Medicine, vol. 334, no. 18, pp. 1150-1155, 1996.

[125] D. D. Waters, E. L. Alderman, J. Hsia et al., "Effects of hormone replacement therapy and antioxidant vitamin supplements on coronary atherosclerosis in postmenopausal women: a randomized controlled trial," Journal of the American Medical Association, vol. 288, no. 19, pp. 2432-2440, 2002.

[126] S. Yusuf, G. Dagenais, J. Pogue, J. Bosch, and P. Sleight, "Vitamin E supplementation and cardiovascular events in highrisk patients. the heart outcomes prevention evaluation study investigators," New England Journal of Medicine, vol. 342, pp. 154-160, 2000.

[127] Z. Ye and H. Song, "Antioxidant vitamins intake and the risk of coronary heart disease: meta-analysis of cohort studies," European Journal of Cardiovascular Prevention and Rehabilitation, vol. 15, no. 1, pp. 26-34, 2008.

[128] D. P. Vivekananthan, M. S. Penn, S. K. Sapp, A. Hsu, and E. J. Topol, "Use of antioxidant vitamins for the prevention of cardiovascular disease: meta-analysis of randomised trials," The Lancet, vol. 361, pp. 2017-2023, 2004.

[129] S. R. Steinhubl, "Why have antioxidants failed in clinical trials?" American Journal of Cardiology, vol. 101, no. 10, pp. S14-S19, 2008. 
[130] O. Ziouzenkova, L. Asatryan, C. Tetta, M. L. Wratten, J. Hwang, and A. Sevanian, "Oxidative stress during ex vivo hemodialysis of blood is decreased by a novel hemolipodialysis procedure utilizing antioxidants," Free Radical Biology and Medicine, vol. 33, no. 2, pp. 248-258, 2002.

[131] A. Sevanian and L. Asatryan, "LDL modification during hemodialysis markers for oxidative stress," Contributions to Nephrology, vol. 137, pp. 386-395, 2002.

[132] L. A. Calò, A. Naso, E. Pagnin et al., "Vitamin E-coated dialyzers reduce oxidative stress related proteins and markers in hemodialysis-a molecular biological approach," Clinical Nephrology, vol. 62, no. 5, pp. 355-361, 2004.

[133] L. A. Calò, A. Naso, A. D’Angelo et al., "Molecular biologybased assessment of vitamin E-coated dialyzer effects on oxidative stress, inflammation, and vascular remodeling," Artificial Organs, vol. 35, no. 2, pp. E33-E39, 2011.

[134] A. P. Levy, "Application of pharmacogenomics in the prevention of diabetic cardiovascular disease: mechanistic basis and clinical evidence for utilization of the haptoglobin genotype in determining benefit from antioxidant therapy," Pharmacology and Therapeutics, vol. 112, no. 2, pp. 501-512, 2006.

[135] S. Blum, U. Milman, C. Shapira et al., "Dual therapy with statins and antioxidants is superior to statins alone in decreasing the risk of cardiovascular disease in a subgroup of middle-aged individuals with both diabetes mellitus and the haptoglobin 22 genotype," Arteriosclerosis, Thrombosis, and Vascular Biology, vol. 28, no. 3, pp. e18-e20, 2008. 


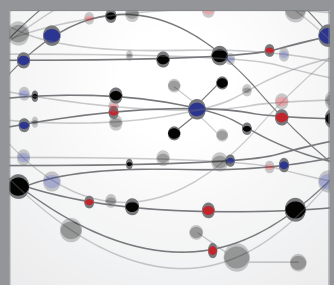

The Scientific World Journal
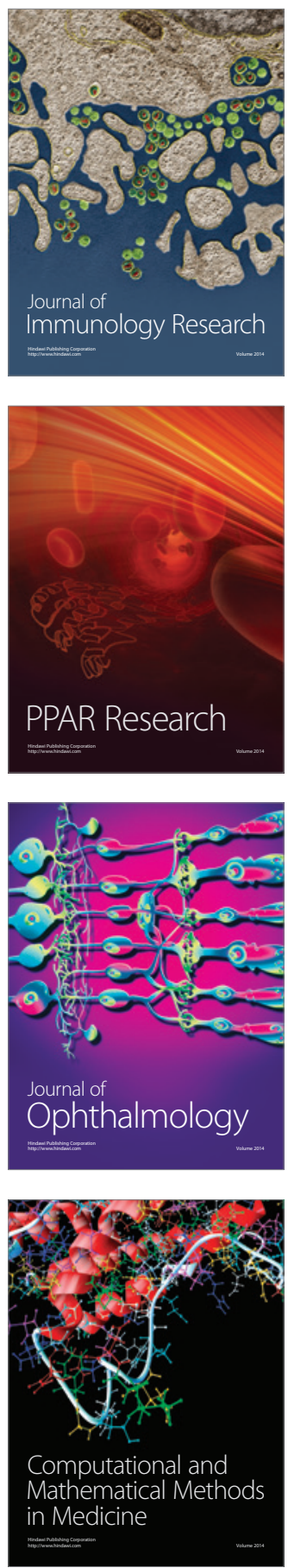

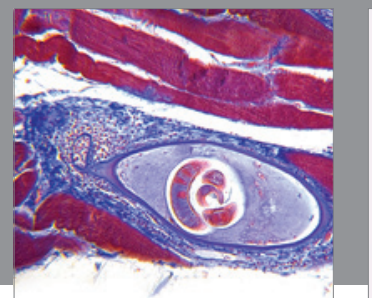

Gastroenterology

Research and Practice
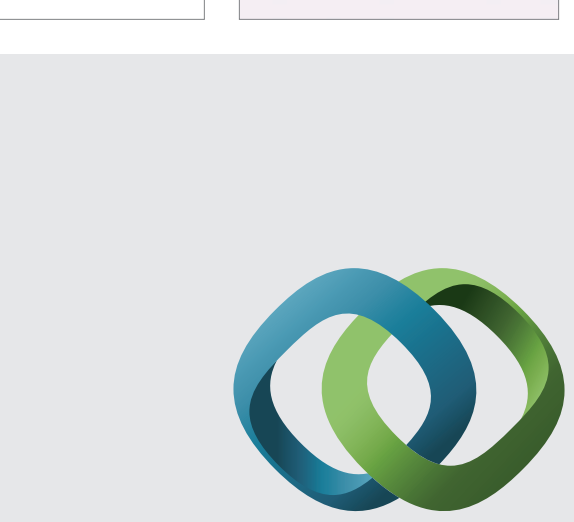

\section{Hindawi}

Submit your manuscripts at

http://www.hindawi.com
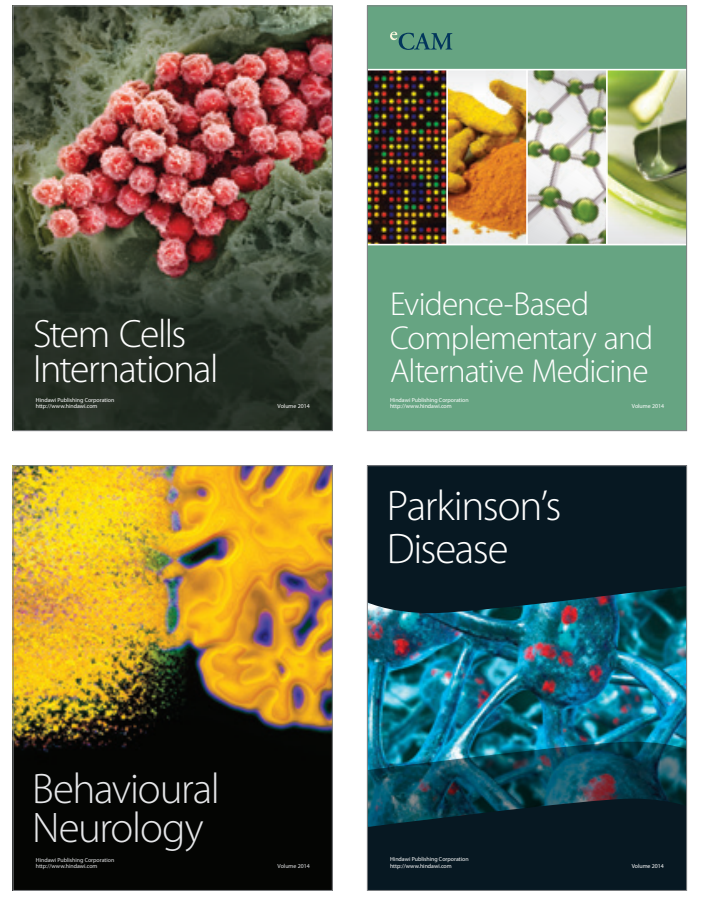
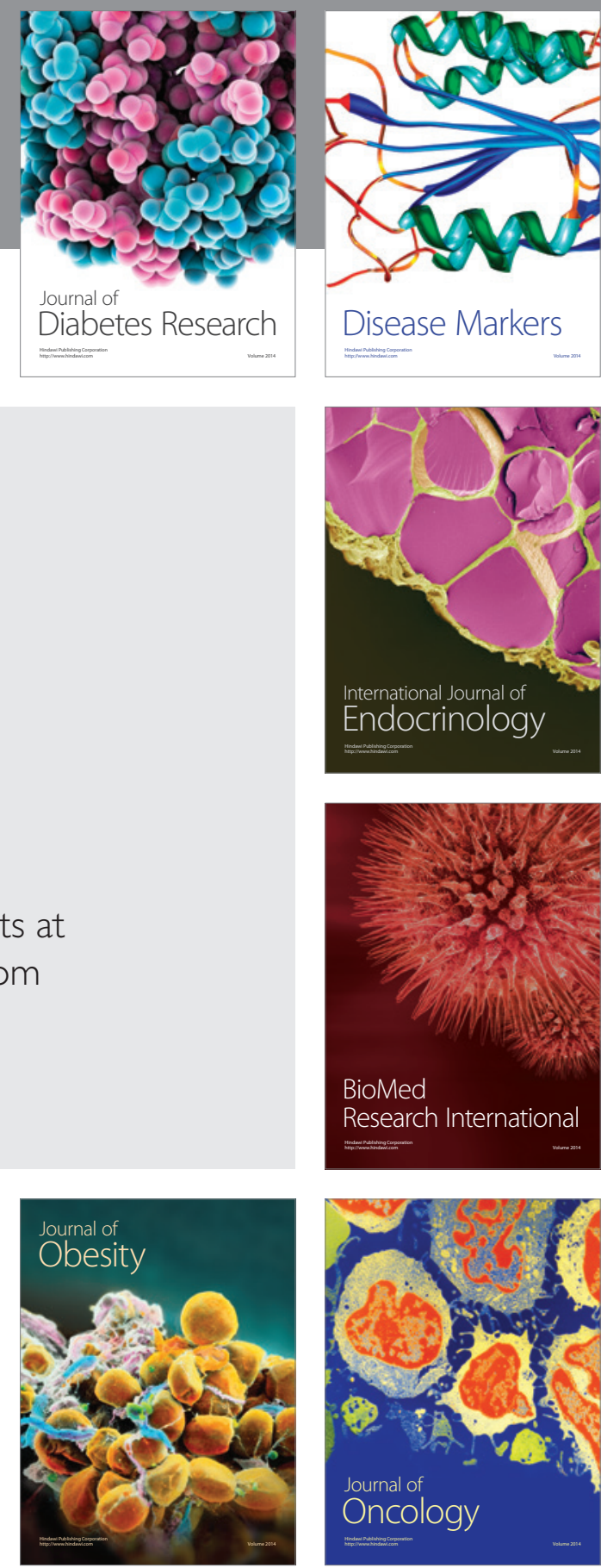

Disease Markers
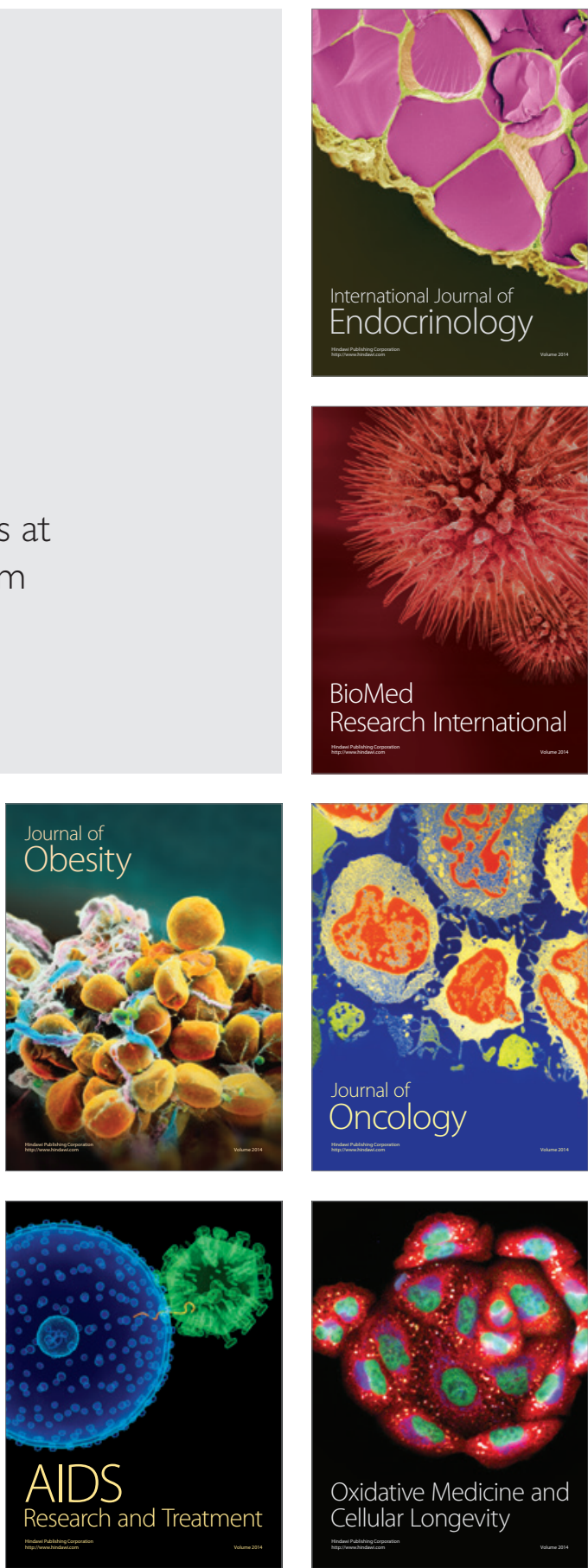